Tropical Journal of Pharmaceutical Research March 2021; 20 (3): 445-451

ISSN: $1596-5996$ (print); 1596-9827 (electronic)

(C) Pharmacotherapy Group, Faculty of Pharmacy, University of Benin, Benin City, 300001 Nigeria.

Available online at http://www.tjpr.org

Original Research Article

http://dx.doi.org/10.4314/tjpr.v20i3.1

\title{
LINC-ROR regulates myocardial ischemia/reperfusion injury via targeting of miR-129-5p/Hook3 axis
}

\author{
Yan Chen ${ }^{1}$, Qianqian Guan², Xiuyuan Feng ${ }^{1 *}$, Xuejun Jiang ${ }^{3}$, Guang $\mathbf{X u}^{1}$, \\ Hongwei Hou', Bike ${ }^{1}$ \\ ${ }^{1}$ Department of Cardiology, Ezhou Central Hospital, Ezhou, ${ }^{2}$ Department of Cardiology, Puren Hospital of Wuhan, ${ }^{3}$ Department \\ of Cardiology, Hubei Provincial People's Hospital, Wuhan, Hubei Province 43000, China
}

*For correspondence: Email: fengxiuyuan666@163.com; Tel: +86-27-60660503

Sent for review: 3 December 2020

Revised accepted: 28 February 2021

\begin{abstract}
Purpose: To investigate the underlying mechanism of long intergenic non-protein coding RNA (LINC$R O R$ ) in myocardial ischemia/reperfusion (I/R) injury.

Methods: Rat H9C2 cells were used to establish the model of hypoxia/reoxygenation (H/R). Cell viability was measured using 3-(4,5-dimethylthiazol-2-yl)-2,5-diphenyltetrazolium bromide (MTT) method. The proportion of apoptotic H9C2 cells was evaluated using flow cytometry. Luciferase reporter experiments and RNA pull-down assays were used to determine the relationships among LINC-ROR, miR-129-5p, and hook microtubule tethering protein 3 (Hook3), while QRT-PCR and western blots were used to investigate the relationship between LINC-ROR and the expression and activation of Hook3 and proteins of the PI3KJAKT/mTOR pathway.

Results: LINC-ROR was elevated under H/R stimulation. The viability of H9C2 cells decreased, and cell apoptosis was induced after $H / R$ treatment. These latter effects were abrogated by the downregulation of LINC-ROR. Luciferase reporter results and RNA pull-down showed that LINC-ROR served as the miR-129-5p sponge, and miR-129-5p was bound to Hook3 RNA directly. Moreover, overexpression of LINC-ROR increased Hook3 protein level by serving as miR-129-5p sponge. Furthermore, the overexpression of LINC-ROR activated PI3KJAkt/mTOR pathway by regulating Hook3. Conclusion: LINC-ROR increases in cardiomyocytes with $H / R$ injury. Down-regulation of LINC-ROR alleviates myocardial I/R injury via miR-129-5p/Hook3 axis. Therefore, LINC-ROR is a potential therapeutic target for myocardial I/R injury.
\end{abstract}

Keywords: LINC-ROR, miR-129-5p, Hook3, Ischemia/reperfusion injury, Hypoxia/reoxygenation

This is an Open Access article that uses a fund-ing model which does not charge readers or their institutions for access and distributed under the terms of the Creative Commons Attribution License (http://creativecommons.org/licenses/by/4.0) and the Budapest Open Access Initiative (http://www.budapestopenaccessinitiative.org/read), which permit unrestricted use, distribution, and reproduction in any medium, provided the original work is properly credited.

Tropical Journal of Pharmaceutical Research is indexed by Science Citation Index (SciSearch), Scopus, International Pharmaceutical Abstract, Chemical Abstracts, Embase, Index Copernicus, EBSCO, African Index Medicus, JournalSeek, Journal Citation Reports/Science Edition, Directory of Open Access Journals (DOAJ), African Journal Online, Bioline International, Open-J-Gate and Pharmacy Abstracts

\section{INTRODUCTION}

Myocardial ischemia/reperfusion $(\mathrm{I} / \mathrm{R})$ is a clinical challenge related to thrombolytic therapy and coronary intervention surgery [1]. During $\mathrm{I} / \mathrm{R}$ injury, inflammation and cardiomyocyte apoptosis is triggered, and myocardial injury is induced through increased production of cytokines and oxygen free radicals [2,3]. Myocardial $\mathrm{l} / \mathrm{R}$ injury usually leads to systolic cardiac dysregulation, arrhythmias, heart failure, and myocardial infarction [1,4]. Furthermore, myocardial $\quad I / R$ 
injury is a leading cause of death related to coronary heart disease [5]. Therefore, it is essential to investigate the molecular mechanism of myocardial I/R injury and explore potential therapeutic strategies. Long non-coding RNAs (IncRNAs) are endogenous non-coding RNA with lengths over 200 nucleotides [6]. Although IncRNAs do not encode proteins, it has been found that IncRNAs play an essential regulatory role in many diseases (including cardiovascular diseases) through functioning as sponges of miRNAs [7]. For example, IncRNA Hox transcript antisense intergenic RNA (HOTAIR) is reported to protect against myocardial infarction through negatively regulating the expression of miR519d-3p [8]. LncRNA ZNFX1 antisense RNA 1 (ZFAS1) and CDR1AS were differentially expressed in acute myocardial infarction patients, indicating that IncRNA ZFAS1 and CDR1AS might be biomarkers of acute myocardial infarction [9]. In myocardial I/R injury, IncRNA urothelial cancer associated 1 (UCA1) regulates cardiomyocyte apoptosis through sponging miR-143 [10].

Previous studies have reported the role of long intergenic non-protein coding RNA (LINC-ROR) in cardiovascular diseases [11,12]. Jiang et al found that LINC-ROR was elevated in hypertrophic heart and cardiomyocytes, and the hypertrophic response was alleviated after the downregulation of LINC-ROR [11]. In addition, LINC-ROR levels were increased in I/R patients and hypoxia/reoxygenation $(\mathrm{H} / \mathrm{R})$ cardiomyocytes. Elevated LINC-ROR decreased cell viability and increased apoptosis, thereby aggravating myocardial I/R injury [12]. Nevertheless, the underlying mechanism of LINC-ROR in myocardial $\mathrm{I} / \mathrm{R}$ injury remains unknown.

Therefore, the objective of this study was to investigate the underlying mechanism of LINCROR modulation of myocardial I/R injury and search for promising therapeutic targets.

\section{EXPERIMENTAL}

Cell culture and $H / R$ injury model establishment

Rat cardiomyocytes (H9C2 cells) were acquired from the American Type Culture Collection
(ATCC) and cultured using Dulbecco's Modified Eagle Medium (DMEM) plus $10 \%$ fetal bovine serum (FBS) (Gibco, Carlsbad, CA, USA) at 37 ${ }^{\circ} \mathrm{C}$ cell incubator with $5 \% \mathrm{CO}_{2}$. To establish the H/R model, H9C2 cells were maintained in serum-free and sugar-free medium in a $37{ }^{\circ} \mathrm{C}$ cell incubator with $94 \% \mathrm{~N}_{2}, 1 \% \mathrm{O}_{2}$, and $5 \% \mathrm{CO}_{2}$ for $24 \mathrm{~h}$, following by transferring to DMEM plus $10 \%$ FBS and maintained in a $37^{\circ} \mathrm{C}, 5 \% \mathrm{CO}_{2}$ atmosphere for $3 \mathrm{~h}$.

\section{Quantitative real-time polymerase chain reaction (qRT-PCR)}

Total RNA in $\mathrm{H} 9 \mathrm{C} 2$ cells was extracted using TRIzol (Sigma, St Louis, MO, USA). Complementary DNA (cDNA) was produced utilizing Mir-X miRNA First-Strand Synthesis Kit (Takara, Beijing, China), and qPCR was conducted using SYBR ${ }^{\mathrm{TM}}$ Green PCR Master Mix (Thermo Fisher Scientific, Waltham, MA, USA). The primers for LINC-ROR, miR-129-5p, and Hook3 are shown in Table 1. $\beta$-actin and U6 were used as reference genes. Data analysis were conducted by $2^{-\Delta \Delta C t}$.

\section{Cell transfection}

The negative control of shRNA, shRNA of LINCROR, LINC-ROR overexpressed plasmid, and shRNA of Hook3 were produced by GenePharma (GenePharma, Shanghai, China). H9C2 cells were plated into 6-well plates at a density of $5 \times 10^{5}$ per well and cultured for $24 \mathrm{~h}$. Then, H9C2 cells were transfected with shRNA negative control, shRNA of LINC-ROR, LINCROR overexpressed plasmid, or shRNA of Hook3 utilizing Lipofectamine 3000 (Thermo Scientific, Waltham, MA, USA) following the manufacturer's protocol. After $48 \mathrm{~h}$, the $\mathrm{H} 9 \mathrm{C} 2$ cells were used for subsequent experiments.

\section{3-(4,5-Dimethylthiazol-2-yl)-2,5- diphenyltetrazolium bromide (MTT) assay}

H9C2 cells were seeded into 96-well plates at 2 $\times 10^{4}$ cells per well and maintained at $37^{\circ} \mathrm{C}$, in a $5 \% \mathrm{CO}_{2}$ atmosphere for $24 \mathrm{~h}$. Then, $10 \mu \mathrm{L}$ of MTT (Sigma-Aldrich, St. Louis, MO, USA) was added to each well. The cells were maintained in a $37{ }^{\circ} \mathrm{C}$ cell incubator with $5 \% \mathrm{CO}_{2}$ for $4 \mathrm{~h}$.

Table 1: Sequences of the primers for PCR

\begin{tabular}{lll}
\hline Gene name & Forward & Reverse \\
\hline LINC-ROR & 5'-AAGGGTGTGAACCTTCCTGG-3' & 5'-GCTCGACCCCAAACTTCAGA-3' \\
miR-129-5p & 5'-ACCCAGTGCGATTTGTCA-3' & 5'-ACTGTACTGGAAGATGGACC-3' \\
Hook3 & 5'-TTCGGCAACAGAATGATGAAC-3' & 5'-AGTTAAGAAAGGCCAACGCAG-3' \\
\hline
\end{tabular}


Subsequently, $100 \mu \mathrm{L}$ DMSO was added to $\mathrm{H} 9 \mathrm{C} 2$ cells to dissolve crystallization. Cell viability was calculated based on the absorbance at $490 \mathrm{~nm}$.

\section{Flow cytometry}

H9C2 cells were collected and treated using phosphate-buffered saline (PBS). Cells were resuspended using $1 \times$ binding buffer after centrifugation was performed. The cells were then treated with $5 \mu \mathrm{L}$ of Annexin V-FITC and 10 $\mu \mathrm{L}$ of propidium iodide (PI) (BD Biosciences, San Jose, CA, USA) in the dark for $15 \mathrm{~min}$, following by detection of the proportion of apoptotic cells through flow cytometry (BD, San Jose, CA, USA).

\section{Luciferase assay}

The wild and mutant LINC-ROR and wild and mutant 3'-UTR of Hook3 were cloned into the pGL3 vector (Promega, Madison, WI, USA). The wild LINC-ROR, mutant LINC-ROR, wild Hook3 3'-UTR, mutant Hook3 3'-UTR, control mimics, or miR-129-5p mimics were transfected into H9C2 cells. After $48 \mathrm{~h}$, luciferase activity was determined using a microplate reader. The relative luciferase activity was measured after normalization.

\section{RNA pull-down assay}

Biotinylated-LINC-ROR and biotinylated negative control (Bio-NC) probes were produced in RiboBio (Guangzhou, China). The LINC-ROR probe was then incubated with Streptavidin C-1 beads (Thermo Fisher Scientific, Waltham, MA, USA) at $25{ }^{\circ} \mathrm{C}$ for $2 \mathrm{~h}$ to produce probe-coated beads. The cell lysates of $\mathrm{H} 9 \mathrm{C} 2$ cells were harvested and mixed with the beads for $12 \mathrm{~h}$ at 4 ${ }^{\circ} \mathrm{C}$. After washing with wash/binding buffer, the miRNA adsorbed on the beads was eluted and quantitated using qRT-PCR assay.

\section{Western blotting}

Total proteins in $\mathrm{H} 9 \mathrm{C} 2$ cells were isolated using RIPA (Thermo Scientific, Waltham, MA, USA) and quantified using BCA methods. Proteins were separated by SDS-PAGE gels, transferred onto PVDF membrane, then blocked for $1 \mathrm{~h}$ using $4 \%$ skim milk, followed by probing with anti-Hook3 (1:500), p-AKT (1: 500), AKT (1: $500), p-m T O R(1: 500), \operatorname{mTOR}(1: 500)$, and $\beta$ actin (1 : 2000) antibodies (Abcam, Cambridge, UK) at $4{ }^{\circ} \mathrm{C}$ for $12 \mathrm{~h}$. The membranes were further incubated with IgG H\&L (HRP) antibody (Abcam, Cambridge, UK). $\beta$-actin was designated as the reference protein. The bands were visualized using ECL Ultra Western HRP Substrate (Sigma, St Louis, MO, USA) and then quantified using Image $\mathrm{J}$ software.

\section{Statistical analysis}

Statistical analysis was conducted utilizing SPSS Statistics (version 22.0, Chicago, IL, USA). Data are shown as mean \pm standard deviation (SD). Group differences were compared using Student's $t$-test and one-way ANOVA with LSD post hoc test. All experiments were performed at least 3 times. The $p<0.05$ was identified as statistically significant.

\section{RESULTS}

\section{LINC-ROR knockdown alleviates H/R-induced cardiomyocyte injury}

To determine the influence of LINC-ROR on H/Rinduced cardiomyocyte injury, the expression of LINC-ROR in H9C2 cells treated using H/R was determined using qRT-PCR. Long intergenic non-protein coding RNA (LINC-ROR) was elevated in $\mathrm{H} 9 \mathrm{C} 2$ cells with $\mathrm{H} / \mathrm{R}$ treatment $(p<$ 0.01 , Figure $1 \mathrm{~A}$ ). Then, knockdown of LINCROR was performed and verified using qRTPCR. Two shRNAs of LINC-ROR significantly down-regulated LINC-ROR expression $(p<0.05$, Figure $1 \mathrm{~A}$ ). Because the shRNA 1 of LINC-ROR knocked down LINC-ROR expression with greater efficiency than shRNA 2 of LINC-ROR (Figure $1 \mathrm{~A}$ ), shRNA 1 of LINC-ROR was used for subsequent experiments. The $\mathrm{H} 9 \mathrm{C} 2$ cells treated using $H / R$ presented decreased cell viability $(p<0.01)$, while transfection with shRNA of LINC-ROR to knock down LINC-ROR increased cell viability ( $p<0.01$, Figure $1 \mathrm{~B}$ ). Furthermore, H/R injury induced $\mathrm{H} 9 \mathrm{C} 2$ cell apoptosis ( $p<0.01$, Figure $1 \mathrm{C}$ ). However, knockdown of LINC-ROR decreased the proportion of apoptotic $\mathrm{H} 9 \mathrm{C} 2$ cells induced by H/R $(p<0.01$, Figure $1 \mathrm{C})$. Thus LINC-ROR knockdown reduced $\mathrm{H} / \mathrm{R}$-induced cardiomyocyte injury.

\section{LINC-ROR acts as miR-129-5p sponge}

LncRNAs were demonstrated to regulate disease development through modulating gene expression by acting as miRNA sponges. Hence, LINC-ROR might alleviate H/R-induced cardiomyocyte injury by acting as miRNA sponges. Bioinformatics analysis found that LINC-ROR might bind to miR-129-5p. The sequence of the potential sites by which LINCROR might target miR-129-5p is shown in Figure 2 A. To confirm whether LINC-ROR bound to miR-129-5p, a luciferase reporter experiment 
was performed. Results showed that miR-129-5p mimics remarkably reduced the activity of wild LINC-ROR reporter $(p<0.01)$ but did not affect the mutant LINC-ROR reporter (Figure $2 \mathrm{~B}$ ). Besides, RNA knock-down results showed higher enrichment of miR-129-5p by LINC-ROR probe ( $p<0.01$, Figure $2 \mathrm{C}$ ). Furthermore, miR-129-5p in $\mathrm{H} 9 \mathrm{C} 2$ cells treated by $\mathrm{H} / \mathrm{R}$ was significantly decreased $(p<0.01)$, which was partly reversed after LINC-ROR knockdown $(p<0.01$, Figure 2 D). Hence, LINC-ROR serves as the miR-129-5p sponge.

\section{LINC-ROR promotes Hook3 expression by functioning as the sponge of $\mathrm{miR}-129-5 p$}

The target genes of miR-129-5p were investigated using TargetScan (http://www.targetscan.org). Hook3 was a putative target of miR-129-5p, and the sequence of the potential binding region are presented in Figure $3 \mathrm{~A}$. Luciferase reporter experiments demonstrated that miR-129-5p mimics significantly decreased the activity of wild 3'-UTR of Hook3 reporter $(p<0.01)$, but did not affect the mutant 3'-UTR of Hook3 reporter (Figure 3 B). Besides, miR-129-5p mimics significantly repressed Hook3 level $(p<0.01)$, and miR-129$5 p$ inhibitor increased Hook3 levels $(p<0.01$, Figure $3 \mathrm{C}$ ). Furthermore, upregulated LINCROR promoted Hook3 expression ( $p<0.01$ ), which was abolished by miR-129-5p mimics ( $p<$ 0.01 , Figure $3 \mathrm{D})$. Hence, these results suggest that LINC-ROR promotes Hook3 expression through serving as the miR-129-5p sponge.

A
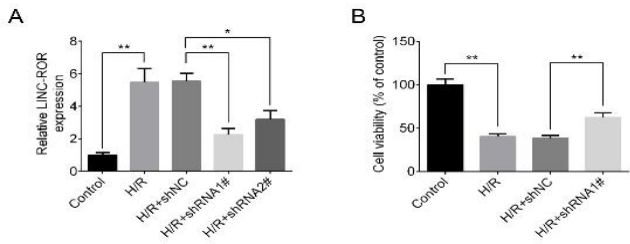

C

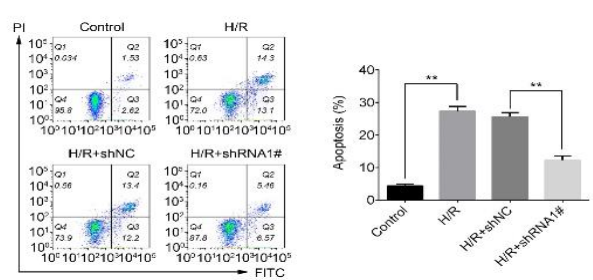

Figure 1: Down-regulation of LINC-ROR alleviates $\mathrm{H} / \mathrm{R}$-induced cardiomyocyte injury. A. The expression of LINC-ROR was determined using qRT-PCR in H9C2 cells treated with $H / R$ and transfected with shRNA of LINC-ROR. B. Cell viability of H/R-treated $\mathrm{H} 9 \mathrm{C} 2$ cells with down-regulation of LINC-ROR was determined using MTT assay. C. Apoptosis of H/Rtreated $\mathrm{H} 9 \mathrm{C} 2$ cells with down-regulation of LINC-ROR was evaluated using flow cytometry. ${ }^{*} P<0.05 ;{ }^{* *} p<$ 0.01

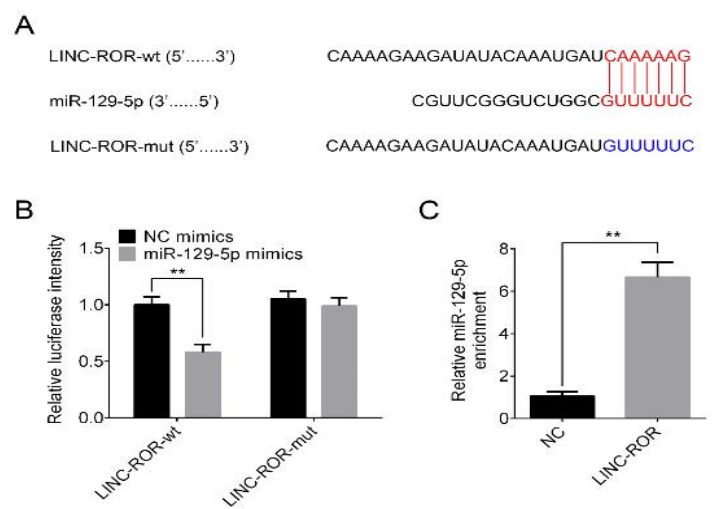

D

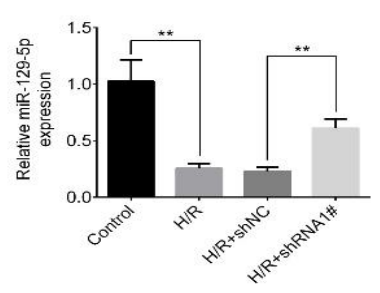

Figure 2: LINC-ROR acts as a sponge for miR-129$5 p$. A. The sequence of the potential binding sites of LINC-ROR and miR-129-5p are presented. B. Luciferase assay was used to investigate the relationship between LINC-ROR and miR-129-5p. C. RNA knock-down assay was performed to study whether LINC-ROR bound to miR-129-5p. D. QRTPCR was used to determine the expression of miR129-5p in H/R-treated H9C2 cells with the downregulation of LINC-ROR. ${ }^{* *} P<0.01$
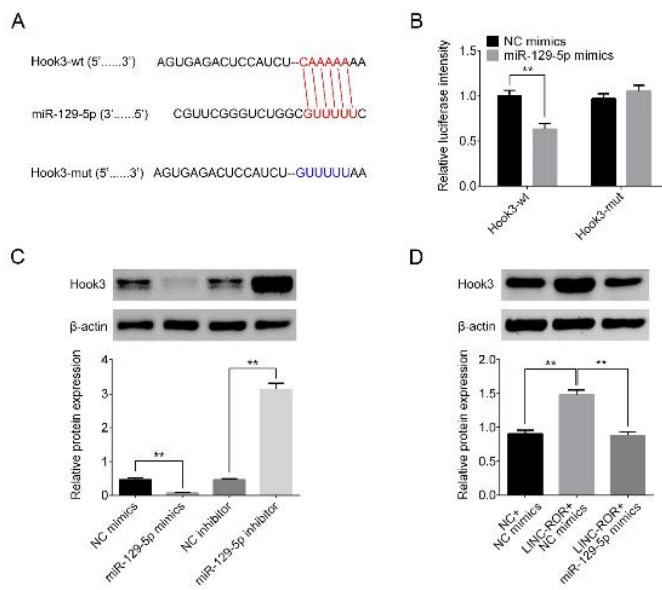

Figure 3: LINC-ROR regulates Hook3 expression via functioning as the sponge for miR-129-5p. A. The sequence of the potential binding sites of miR-129-5p and Hook3 are presented. B. Luciferase assays were performed to explore the relationship between miR$129-5 p$ and Hook3. C. The protein level of HooK3 in H9C2 cells transfected with miR-129-5p mimics and inhibitor was determined using western blot. D. The protein level of HooK3 in $\mathrm{H} 9 \mathrm{C} 2$ cells transfected with LINC-ROR overexpressing plasmid and miR-129-5p mimics was detected by western blot. ${ }^{* \star} P<0.01$ 


\section{LINC-ROR regulates $H / R$ injury and PI3K/Akt/mTOR pathway activation via regulating Hook3}

To investigate the mechanism underlying the effect of LINC-ROR on regulation of H/R injury, the H/R-induced $\mathrm{H} 9 \mathrm{C} 2$ cells were transfected with LINC-ROR overexpression plasmid and shRNA of Hook3. The MTT results showed that the viability of $\mathrm{H} 9 \mathrm{C} 2$ cells overexpressing LINCROR was decreased ( $p<0.01$ ), but this effect was reversed by decreased Hook3 expression ( $p$ $<0.01$, Figure $4 \mathrm{~A}$ ). Furthermore, cell apoptosis was induced by overexpression of LINC-ROR ( $p$ $<0.01$ ), but this effect was also reversed by decreased Hook3 expression $(p<0.01$, Figure 4 $B)$. Moreover, overexpression of LINC-ROR increased the expression of Hook3 $(p<0.01)-$ an effect that was reversed by decreased Hook3 expression $(p<0.01$, Figure 4 C). Overexpression of LINC-ROR also increased $p$ AKT/AKT and $p-m T O R / T O R$ ratios $(p<0.01)$, but this effect was reversed by the downregulation of Hook3 expression ( $p<0.01$, Figure $4 \mathrm{C})$. Therefore, LINC-ROR regulates H/Rinduced cardiomyocyte injury and the $\mathrm{PI3K} / \mathrm{Akt} / \mathrm{mTOR}$ pathway via regulation of Hook3.

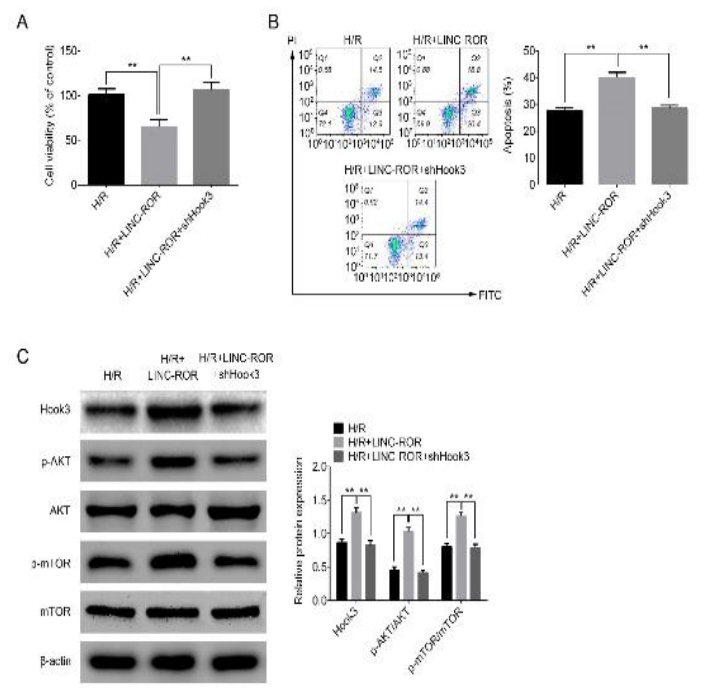

Figure 4: LINC-ROR regulates $\mathrm{PI} 3 \mathrm{~K} / \mathrm{Akt} / \mathrm{mTOR}$ pathway via regulating Hook3. A. MTT assay was conducted to determine the cell viability of H/R-treated H9C2 cells with overexpression of LINC-ROR and down-regulation of Hook3. B. Apoptosis of H/R-treated H9C2 cells with overexpression of LINC-ROR and down-regulation of Hook3 was determined using flow cytometry. C. The protein levels of Hook3, p-AKT, AKT, p-mTOR, and mTOR in H/R-treated H9C2 cells with overexpression of LINC-ROR and downregulation of Hook3 were determined using western blot. ${ }^{* *} P<0.01$

\section{DISCUSSION}

Myocardial ischemia/reperfusion (I/R) is a complicated clinical issue, usually leading to systolic cardiac dysregulation, arrhythmias, heart failure, and myocardial infarction $[1,4]$. Thus, it is essential to study the underlying mechanism of I/R injury and search for prospective curative strategies. Long intergenic non-protein coding RNA (LINC-ROR) regulates myocardial I/R injury, but the potential mechanism of action remains unknown.

To explain the mechanism of LINC-ROR in myocardial I/R injury, an $H / R$ cell model was established. Long intergenic non-protein coding RNA (LINC-ROR) was elevated in $\mathrm{H} 9 \mathrm{C} 2$ cells after $H / R$ treatment, which is consistent with previous work [12] reporting that LINC-ROR was up-regulated in $I / R$ patients and $H / R$ cardiomyocytes [12]. After the down-regulation of LINC-ROR in H/R-treated $\mathrm{H} 9 \mathrm{C} 2$ cells, cell viability was increased, and cell apoptosis was decreased. These findings were consistent with a previous study demonstrating that cardiomyocytes' cell viability was suppressed using $H / R$, but that this effect could be reversed by decreasing LINC-ROR expression [12]. Furthermore, Zhang et al reported that knockdown of LINC-ROR decreased cell apoptosis in cardiomyocytes with $\mathrm{H} / \mathrm{R}$ treatment [12]. Nevertheless, whether LINC-ROR regulated IR injury via acting as a competitive endogenous RNA (ceRNA) was not reported in the study conducted by Zhang et al.

LncRNAs usually act as ceRNAs to participate in disease progression via serving as sponges of miRNAs. For instance, Yang et al. reported that LINC-ROR increased the radiotherapy resistance of human colorectal cancer through sponging miR-145 [13]. Thus, potential target miRNAs of LINC-ROR were screened to determine the underlying mechanism whereby LINC-ROR regulates $\mathrm{H} / \mathrm{R}$-induced cardiomyocyte injury. According to the results in this study, LINC-ROR bound to miR-129-5p and negatively regulated miR-129-5p expression. This miRNA has been found to participate in the regulation of cardiovascular diseases [14,15]. Xiao et al showed that miR-129-5p improved cardiac function in the rat chronic heart failure model via targeting HMGB1 [14]. Furthermore, Chen et al showed that miR-129-5p moderated myocardial I/R injury via regulating HMGB1 [15].

In this study, the target of miR-129-5p was also investigated. The results revealed that Hook3 was targeted by miR-129-5p, and miR-129-5p negatively modulated Hook3 expression. 
Interestingly, the expression of Hook3 was positively regulated by LINC-ROR while serving as the sponge of miR-129-5p. Hook3 is an adaptor for microtubule-dependent intracellular vesicle and protein trafficking [16]. Hook3 mediated the beneficial effect of miR-496 on H/R injury [17].

Similarly, results found that LINC-ROR exerted protecting effects on cell viability and cell apoptosis in $\mathrm{H} 9 \mathrm{C} 2$ cells after $\mathrm{H} / \mathrm{R}$ treatment through regulating Hook3 expression in this study. Hence, LINC-ROR promotes Hook3 expression through serving as miR-129-5p sponge and alleviates H/R-induced cardiomyocyte injury via regulating Hook3. Furthermore, results in the study revealed that overexpression of LINC-ROR activated $\mathrm{PI} 3 \mathrm{~K} / \mathrm{Akt} / \mathrm{mTOR}$ pathway via regulating Hook3. Accumulating evidence shows that the $\mathrm{PI}$ KK/Akt/mTOR signaling activation was critical for protecting against myocardial I/R injury [18]. Based on the above evidence, LINC-ROR might regulate the expression of Hook3 via sponging miR-129-5p, thereby prompting PI3K/Akt/mTOR signaling and attenuating $H / R$ injury. Therefore, LINC-ROR was a critical molecule in myocardial $\mathrm{l} / \mathrm{R}$ injury, making it a promising target for myocardial I/R injury therapy.

\section{CONCLUSION}

Long intergenic non-protein coding RNA (LINCROR) increases in cardiomyocytes treated with $H / R$. Down-regulation of LINC-ROR alleviates myocardial I/R injury via the miR-129-5p/Hook3 axis, making LINC-ROR a prospective target for myocardial I/R injury therapy.

\section{DECLARATIONS}

\section{Conflict of interest}

No conflict of interest is associated with this work.

\section{Contribution of authors}

We declare that this work was done by the authors named in this article and all liabilities pertaining to claims relating to the content of this article will be borne by the authors. Yan Chen and Qianqian Guan designed the study and supervised the data collection; Xiouyuan Feng and XuejunJiang prepared the manuscript for publication and reviewed the draft of the manuscript; and Guang $\mathrm{Xu}$, Honwei Hou, and BiKe analyzed and interpreted the data. All authors have read and approved the manuscript.
Yan Chen and Qianqian Guan contributed equally to the work.

\section{Open Access}

This is an Open Access article that uses a funding model which does not charge readers or their institutions for access and distributed under the terms of the Creative Commons Attribution License (http://creativecommons.org/licenses/by/ 4.0) and the Budapest Open Access Initiative (http://www.budapestopenaccessinitiative.org/rea d), which permit unrestricted use, distribution, and reproduction in any medium, provided the original work is properly credited.

\section{REFERENCES}

1. Dhalla NS, Elmoselhi AB, Hata T, Makino N. Status of myocardial antioxidants in ischemia-reperfusion injury. Cardiovasc Res 2000; 47(3): 446-456.

2. Eltzschig HK, Eckle T. Ischemia and reperfusion-from mechanism to translation. Nat Med 2011; 17(11): 1391 1401.

3. Lou $Y, X u B, L i X, X u X$, Chen $X$. Schisandra chinensis extract ameliorates myocardial ischemia/reperfusion injury via TLR4/NF-KB/MyD88 signaling pathway. Trop J Pharm Res 2020; 19(1): 57-62.

4. Yang M, Kong DY, Chen JC. Inhibition of miR-148b ameliorates myocardial ischemia/reperfusion injury via regulation of Wnt/ $\beta$-catenin signaling pathway. J Cell Physiol 2019; 234(10): 17757-17766.

5. Erdal C, Karakülah G, Fermancı E, Kunter I, Silistreli E, Canda $T$, Erdal E, Hepaguslar H. Early biventricular molecular responses to an acute myocardial infarction. Int J Med Sci 2012; 9(1): 74.

6. Huang $Y$. The novel regulatory role of Inc RNA-mi RNA-mRNA axis in cardiovascular diseases. J Cell Mol Med 2018; 22(12): 5768-5775.

7. Li M, Duan L, Li Y, Liu B. Long noncoding RNA/circular noncoding RNA-miRNA-mRNA axes in cardiovascular diseases. Life Sci 2019; 233: 116440.

8. Zhang $D$, Wang $B, M a M, Y u K$, Zhang $Q$, Zhang $X$. InCRNA HOTAIR protects myocardial infarction rat by sponging miR-519d-3p. J Cardiovasc Transl 2019; 12(3): 171-183.

9. Zhang Y, Sun L, Xuan L, Pan Z, Li K, Liu S, Huang Y, Zhao $X$, Huang L, Wang Z. Reciprocal changes of circulating long non-coding RNAs ZFAS1 and CDR1AS predict acute myocardial infarction. Sci Rep 2016; 6: 22384.

10. Yu S-y, Dong B, Zhou S-h, Tang L. LncRNA UCA1 modulates cardiomyocyte apoptosis by targeting miR143 in myocardial ischemia-reperfusion injury. Int $J$ Cardiol 2017; 247(31.

11. Jiang F, Zhou $X$, Huang J. Long non-coding RNA-ROR mediates the reprogramming in cardiac hypertrophy. PLoS One 2016; 11(4): e0152767.

Trop J Pharm Res, March 2021; 20(3): 450 
12. Zhang W, Li Y, Wang P. Long non-coding RNA-ROR aggravates myocardial ischemia/reperfusion injury. Braz J Med Biol Res 2018; 51(6).

13. Yang $P$, Yang $Y$, An $W, X u$ J, Zhang G, Jie J, Zhang Q. The long noncoding RNA-ROR promotes the resistance of radiotherapy for human colorectal cancer cells by targeting the p53/miR-145 pathway. J Gastroen Hepatol 2017; 32(4): 837-845.

14. Xiao N, Zhang J, Chen C, Wan Y, Wang N, Yang J. miR129-5p improves cardiac function in rats with chronic heart failure through targeting HMGB1. Mamm Genome 2019; 30(9-10): 276-288.

15. Chen Z, He D, Mo Q, Xie L, Liang J, Liu L, Fu W. MiR129-5p protects against myocardial ischemiareperfusion injury via targeting HMGB1. Eur Rev Med Pharmaco 2020; 24(8): 4440-4450.
16. Melling N, Harutyunyan L, Hube-Magg C, Kluth M, Simon $R$, Lebok P, Minner S, Tsourlakis MC, Koop C, Graefen M. High-level HOOK3 expression is an independent predictor of poor prognosis associated with genomic instability in prostate Cancer. PLoS One 2015; 10(7): e0134614.

17. Jin Y, Ni S. miR-496 remedies hypoxia reoxygenationinduced H9c2 cardiomyocyte apoptosis via Hook3-targeted PI3k/Akt/mTOR signaling pathway activation. J Cell Biochem 2020; 121(1): 698-712.

18. Li Q, Shen L, Wang Z, Jiang H-P, Liu L-X. Tanshinone IIA protects against myocardial ischemia reperfusion injury by activating the PI3K/Akt/mTOR signaling pathway. Biomed Pharmacother 2016; 84: 106-114. 